\title{
Unilateral Biportal Endoscopic Lumbar Interbody Fusion: A Technical Note and an Outcome Comparison with the Conventional Minimally Invasive Fusion
}

\author{
Asrafi Rizki Gatam' \\ Luthfi Gatam' \\ Harmantya Mahadhipta ${ }^{2}$ \\ Ajiantoro Ajiantoro ${ }^{3}$ \\ Omar Luthfi ${ }^{4}$ \\ Dina Aprilya (iD ${ }^{5}$ \\ 'Orthopaedic Spine Division, Fatmawati \\ General Hospital, Jakarta, Indonesia; \\ ${ }^{2}$ Orthopaedic Spine Division, Tangerang \\ General Hospital, Banten, Indonesia; \\ ${ }^{3}$ Orthopaedic Spine Division, Premier \\ Bintaro Hospital, Banten, Indonesia; \\ ${ }^{4}$ Orthopedic Spine Division, Adhyaksa \\ General Hospital, Jakarta, Indonesia; \\ ${ }^{5}$ Orthopedic and Traumatology \\ Department, Faculty of Medicine \\ Universitas Indonesia, Jakarta, Indonesia
}

\begin{abstract}
Background: In the past few decades, the minimally invasive technique for spine surgery has developed extensively from the scope of decompression until fusion surgeries to reduce damages to the normal anatomical structure. Unilateral biportal endoscopic lumbar interbody fusion (ULIF) is one of the fusion options which is readily available without a sophisticated minimal invasive instrument. Our aim is to introduce ULIF experience in our center and comparing the result with conventional minimally invasive lumbar interbody fusion (MIS-TLIF).
\end{abstract}

Methods: This is a retrospective cohort study of 145 lumbar spondylolisthesis cases that underwent fusion surgery with either ULIF or the conventional MIS-TLIF. All of the patients were observed within a 12-month follow-up period to evaluate the back pain and leg pain Visual Analogue Score (VAS), the Oswestry Disability Index (ODI), the 36-Item Short Form Health Survey (SF-36), and fusion rate.

Results: The leg pain VAS was similarly improved in both groups. ULIF has a significant back pain improvement on direct post operation and at the 3-months follow-up ( $p$ value 0.032 and 0.046 respectively). ULIF group also had a significantly better improvement of ODI scores on the early post-operative period ( $p=0.045$ ). However, both groups similarly showed improvement of ODI score and the SF-36 at the 3-, 6-, and 12- months follow up. Conclusion: Full endoscopic fusion surgery with ULIF offers a comparable long-term outcome and a significantly better back pain VAS reduction in short-term follow up compared to the conventional MIS-TLIF. ULIF, with further improvement, can be the next gold standard in managing degenerative lumbar spine conditions.

Keywords: lumbar spine, degenerative spondylolisthesis, minimally invasive spine surgery, lumbar interbody fusion, unilateral biportal endoscopy, full endoscopic spine surgery, MISS

\section{Introduction}

The lumbar interbody fusion technique has been developed extensively over the last 3 decades. The conventional open surgery, which has been the gold standard since the $1980 \mathrm{~s}$, is slowly being replaced by minimal invasive surgery to achieve better outcomes and patient satisfaction. ${ }^{1}$ The minimally invasive (MIS) technique can reduce the disruption to posterior anatomical structures such as muscles, ligaments and the bone itself. ${ }^{2}$ Spinal decompression using full endoscopic technique has been proven to have many advantages compared to the conventional technique. However, the evidence for the endoscopic-assisted fusion is still lacking., 3,4
Correspondence: Dina Aprilya Orthopedic and Traumatology Department, Faculty of Medicine Universitas Indonesia, Jakarta, Indonesia Email dina.cia.aprilya@gmail.com 
Recently, the full endoscopic lumbar spine fusion has increased in popularity among surgeons, as it provides the ability to better endplate preparation by direct vision to achieve better fusion. ${ }^{5-8}$ There are several techniques for endoscopic-assisted lumbar interbody fusion by either transforaminal or interlaminar approach. The transforaminal approach is done quite similar with uniportal percutaneous endoscopic discectomy (with or without superior articular process [SAP] resection), followed by endplate resection and cage insertion. ${ }^{5,9}$ This technique allows fusion with a stand-alone cage or in combination with pedicle screw insertion. ${ }^{9}$ The problem with this technique is preparation of the endplate, because the endoscope is relatively fixed. Thus, preparing a good fusion-bed is sometimes difficult.

The spinal fusion with the interlaminar approach can be done using either biportal endoscopic or uniportal endoscopic procedure. ${ }^{10,11}$ The endoscopic view of both procedures is quite easy to interpret compared with the transforaminal approach, especially for surgeons who are in the initial learning curve of endoscopic surgery. The biportal technique has advantages of zero investment by using standard arthroscopic lenses at either $0^{\circ}, 30^{\circ}$ or even $70^{\circ}$ angle, more instrument mobility, and a wide viewing angle. Furthermore, open surgery instruments such as forceps, Kerrison punch, and end-plate curettes that are usually available in the hospital. ${ }^{12,13}$ The main disadvantage of this technique is having 2 portals instead of one, which causes more soft tissue injury compared to the uniportal technique.

The fusion rate for endoscopic lumbar interbody fusion remains controversial. Most of the surgeons who have been performing the conventional procedure for decades do not believe in the endoscopic fusion procedure, even though some of the research shows good to excellent fusion within 12 months after the procedure. In this article, we would like to share our technique for unilateral biportal endoscopic lumbar interbody fusion (ULIF) and compare the result with the conventional minimally invasive transforaminal interbody fusion (MIS-TLIF).

\section{Materials and Methods}

This is a retrospective study conducted at 3 public hospitals in Jakarta, Indonesia. From the data collection between January 2016 and April 2020, there were 170 patients who matched the inclusion criteria: Grade 1 or 2 single level degenerative spondylolisthesis with neurological symptoms and mechanical back pain that were treated with interbody fusion procedure. The exclusion criteria were previous spinal instrumentation, spinal tumor, fracture, and infection, were not included into the study. Among those who are eligible for research subject, there were 14 patients who declined to be involved and 11 patients were lost during follow-up. As many as 145 patients were successfully observed for 1 year, this included 73 patients who underwent MIS-TLIF and 72 patients who underwent UBE-TLIF (ULIF).

All subjects underwent conservative management for at least 3 months without clinical improvement. Anteroposterior, lateral and dynamic (flexion and extension) lumbar plain radiograph along with non-contrast lumbar magnetic resonance imaging (MRI) was obtained before the surgery. The radiological series other than MRI were repeated on the 3-, 6-, and 12-month follow-up. The visual analog scale (VAS) for back and leg pain, Oswestry Disability Index (ODI) and 36Item Short Form Health Survey (SF-36) were evaluated before surgery, and at 3-, 6-, and 12-months after surgery. At the end of follow-up (12 months), fusion was evaluated via CT scan examination.

\section{Surgical Techniques for Unilateral Biportal Endoscopic Interbody Fusion}

\section{Position, Anesthesia and Approach}

The patient is prepared in the prone position with general anesthesia. Two bolsters are placed on the chest and the anterior superior iliac spine. This position opens the interlaminar window at the beginning of the procedure by raising the table. Moreover, it also helps to maintain lumbar lordosis by neutralizing the table at the end of procedure while inserting the interbody cage. The general anesthesia provides more comfort for the patients and easier for mean arterial pressure maintenance during surgery. However, regional anesthesia can be used in patients with several comorbidities.

\section{Portal Placement}

The viewing portal and working portal are positioned at the level of pedicle above and below level of fusion (biportal technique). Two $5 \mathrm{~mm}$ transverse incisions are made. For a right-handed person, the portal on the right-hand side is used as the working portal and on the left-hand side is used as the viewing portal. Working space is created subperiosteally using periosteal elevator that is inserted on the working portal and scope trocar are introduced through the viewing 
portal. The triangulation maneuver is completed after the trocar and the periosteal elevator meet on the edge of the lamina. The next step would be irrigating the water from the trocar into the working space. The water should be able to come out of the working portal (Figure 1).

\section{Flavectomy and Foraminectomy}

Using a high-speed burr, inferior edge of the lamina is drilled until the insertion of the flavum ligament, base of spinous process, and the inner cortex of contralateral lamina up to the contralateral facet joint. The purpose of drilling is to thin out and decorticate the outer cortex of the lamina. A combination of the Kerrison punch and chisel is use to complete the laminectomy and foraminectomy. Foraminectomy is begin with removing the inferior articular process (IAP) until seeing base of SAP. The medial part of SAP which is also the roof of lateral recess is also resected using either the Kerrison punch or chisel. The tip of the SAP is removed to create a bigger space on the foraminal area for easier discectomy, end-plate removal and cage insertion.
Like the conventional MIS-TLIF, biportal endoscopic TLIF can achieve a complete neural decompression through laminectomy and facetectomy. After all the bony work is finished, flavectomy is started either with an en bloc resection or piece-by-piece removal using the Kerrison punch (Figure 2). A total flavectomy is not routinely executed. It depends on the clinical symptoms, the severity of flavum thickening and location of the stenosis. The contralateral decompression is started by ipsilateral flavectomy. The ligamentum flavum is detached with the help of the bent probe and then the flavectomy proceed medially passing the midline until reaching the contralateral outer boundary of the spinal canal. Complete decompression would have signs of a pulsating thecal sac, traversing the nerve root and exiting the nerve root.

\section{Discectomy, End-Plate Removal and Cage Insertion}

A radiofrequency probe is used to help the disc exposure. There are usually abundant epidural veins and some adhesion

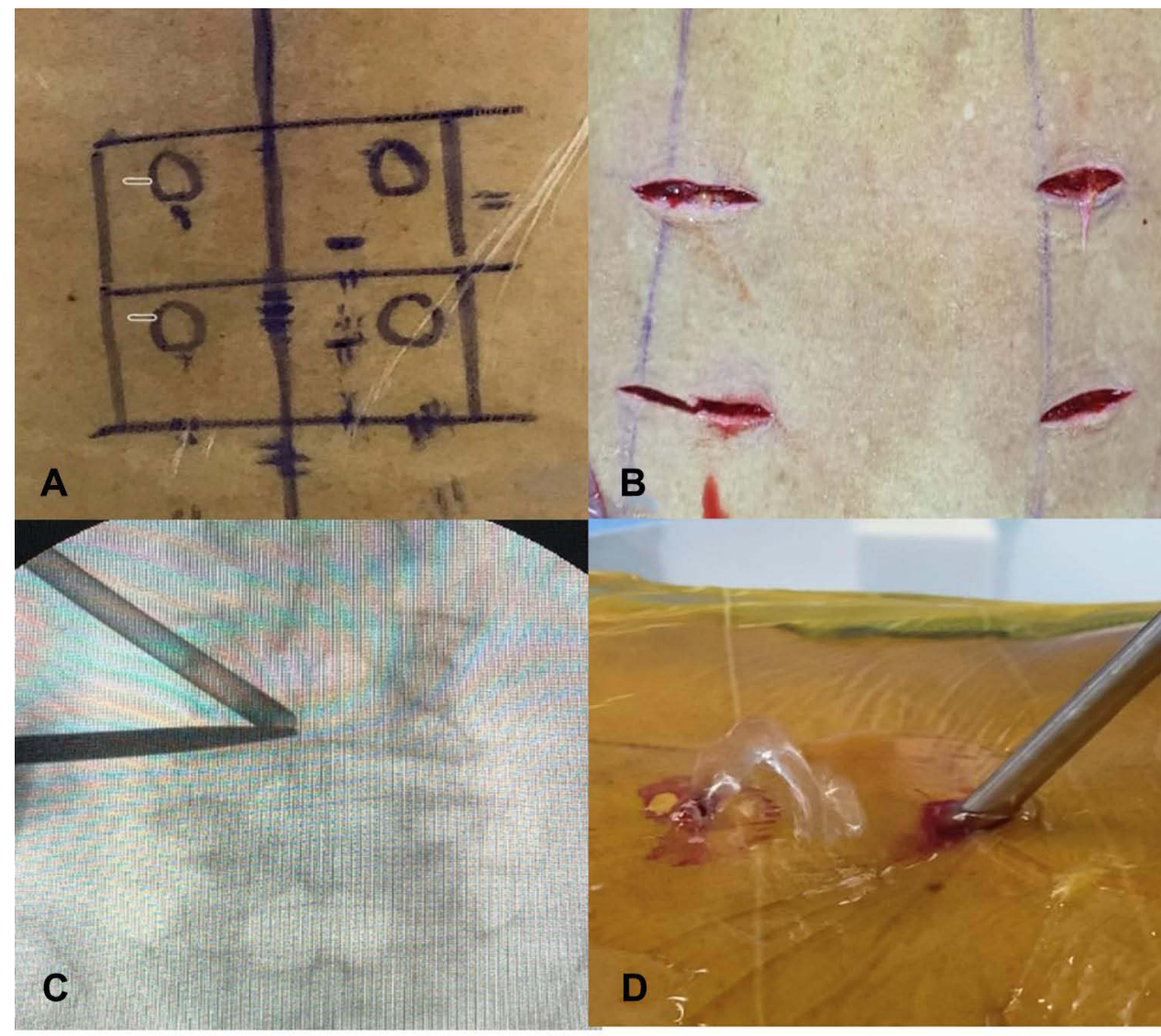

Figure I (A and B) Location of incision at the level of the pedicle. (C) Triangulation of working and viewing portal above the lamina. (D) Waterflow from the working portal. 

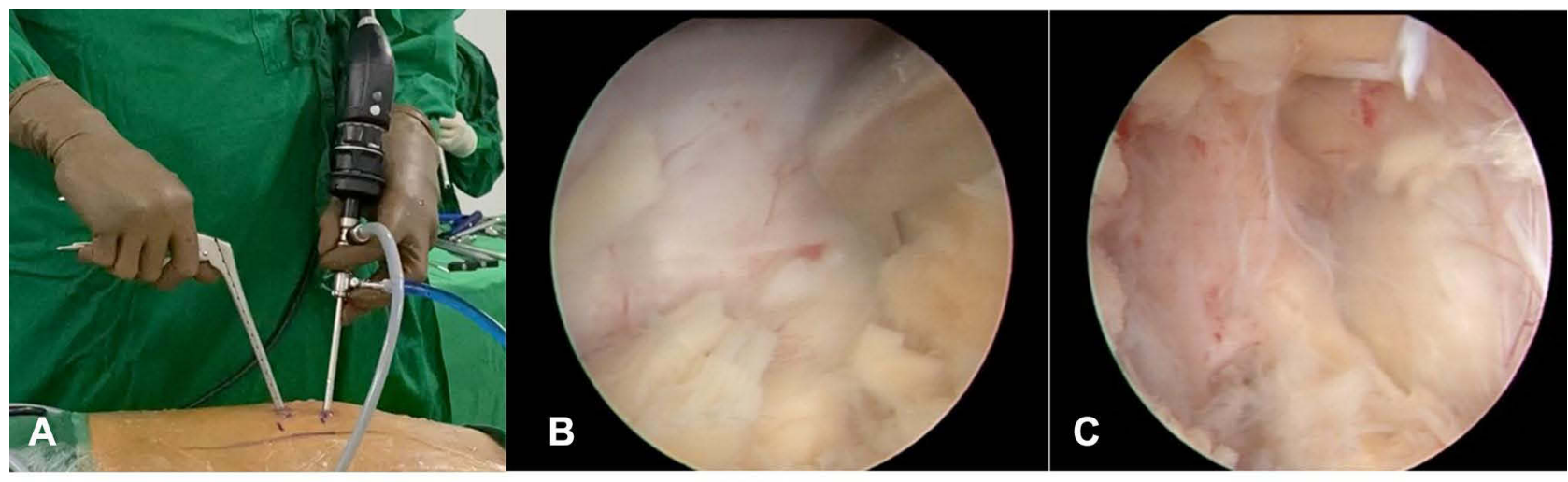

Figure 2 (A) Working position using unilateral biportal endoscopy. (B) Flavectomy piece by piece using Kerrison punch. (C) Contralateral decompression.

of the dura to the disc. The nerve retractor is inserted from the working channel to mobilize the traversing root and the thecal sac medially. Annulotomy is started using radiofrequency probe and continue with discectomy using shaver, curettes, forceps and endplate remover. The endplatepreparation is completed by the evidence of multiple spots of bleeding from the bone. This bone bleeding can only be seen by the aid of endoscopy because the lens can go into the disc space (Figure 3). Cage trial(s) is then inserted through the working channel until it is the appropriate size (Figure 4). Preferably, the insertion of the cage for the right-handed person is done through the right side of the patient, especially on the level L4-5 or L5-S1, because the working portal is located on the rostral side and on these levels the intervertebral space is tilted caudally (Figure 5).

\section{Pedicle Screw Insertion and Listhesis Reduction}

Supplementation pedicle screw fixation are done using percutaneous technique under fluoroscopic guidance
(Figure 6). Several techniques can be done to reduce the listhesis:

1. The rostral pedicle screw is inserted deeper in order to help reduce the listhesis.

2. Patients are held in extension by neutralizing the table.

3. The rod is inserted and a cantilever maneuver is done by tightening the caudal screw before tightening the rostral screw.

All data were calculated using IBM SPSS Statistic 25 with non-parametric statistics using Mann-Whitney $U$ and proportion data using the Fisher exact test. A $p<0.05$ was considered statistically significant.

\section{Results}

All patients had degenerative spondylolisthesis and underwent the interbody procedure either by the conventional MISTLIF or ULIF. Match control was conducted between both

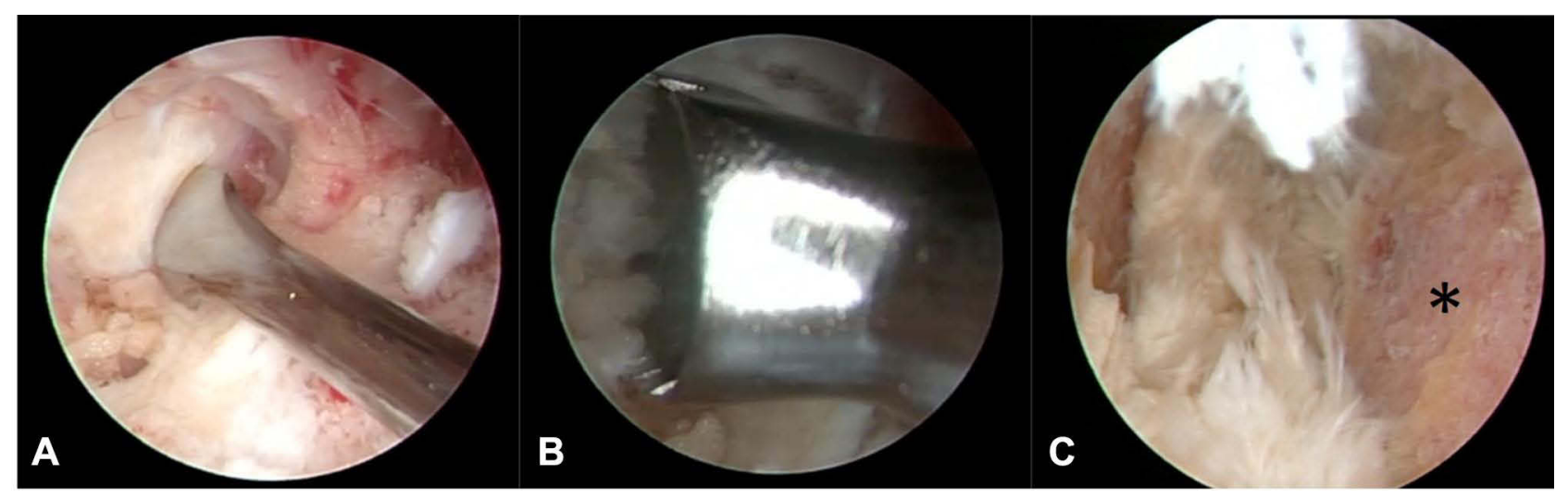

Figure 3 (A) Annulotomy using an annular cutter. (B) Preparation of endplate. (C) Endplate removal completed (asterisk). 


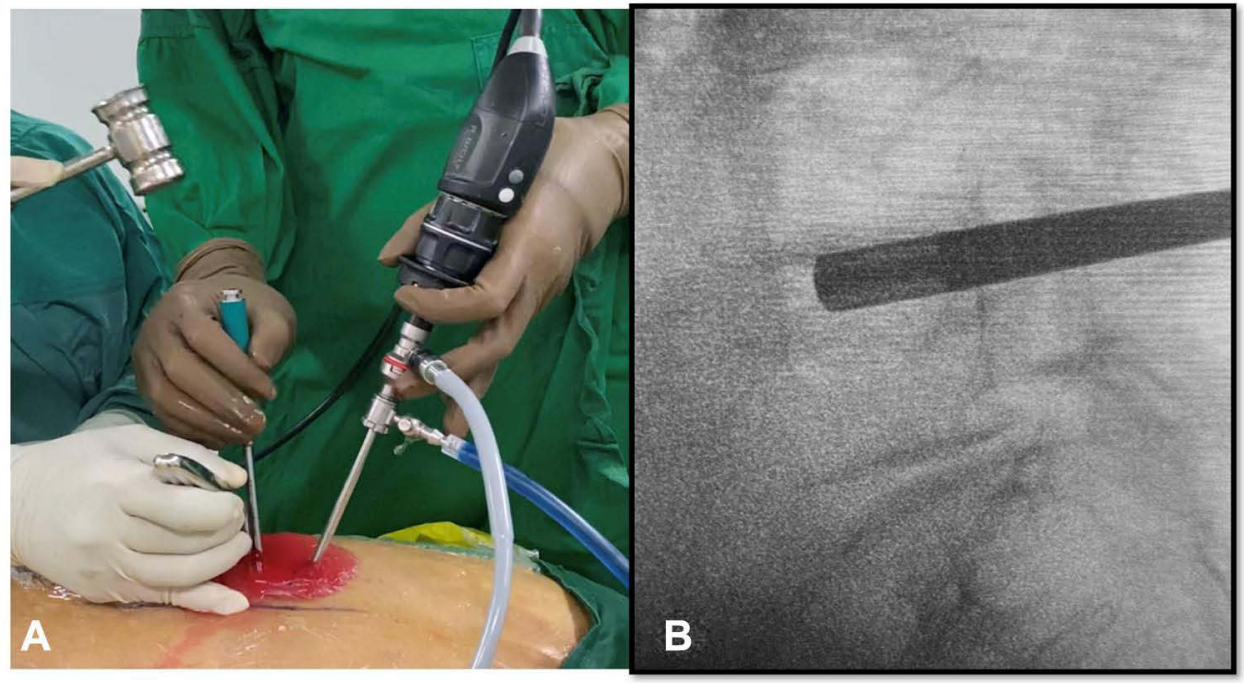

Figure 4 (A) Insertion of reamer under fluoroscopic view. (B) Confirmation on image intensifier.
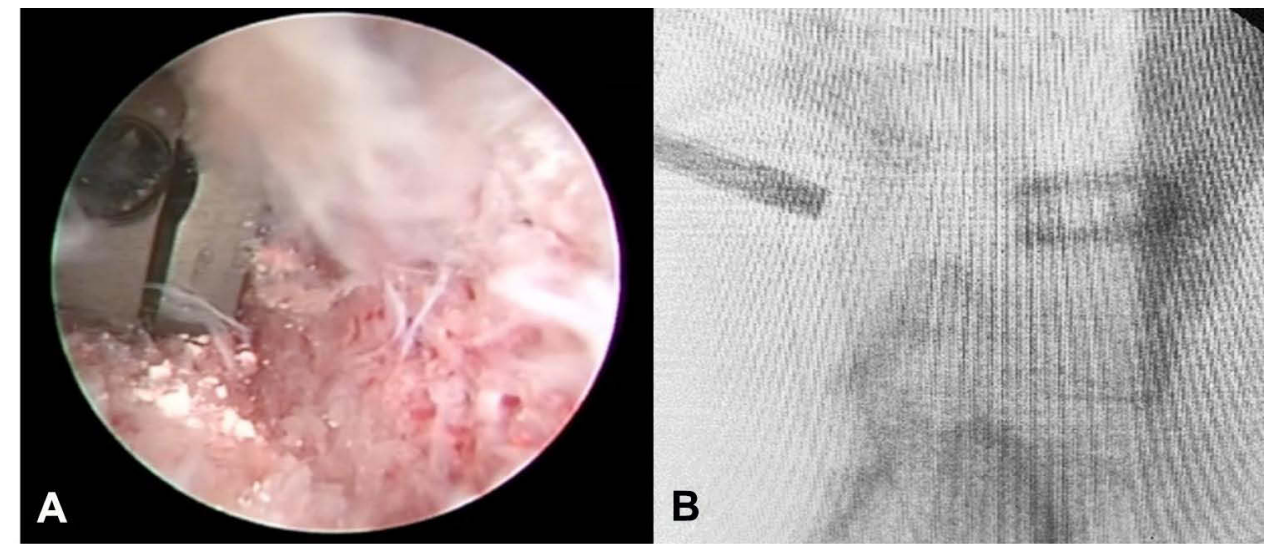

Figure 5 (A) Cage insertion under endoscopic view. (B) Confirmation on image intensifier.

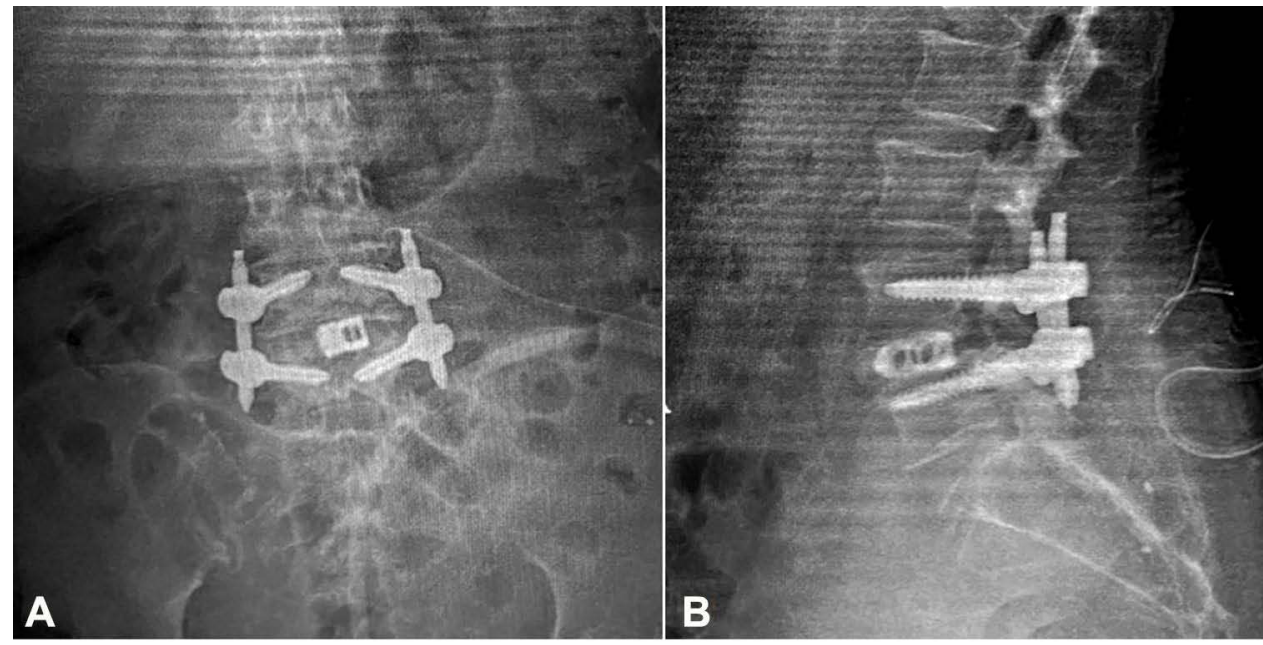

Figure 6 After pedicle screw insertion from AP (A) and lateral (B) view. 
groups in terms of age, sex, body mass index (BMI), operation level and smoking history. The mean age for the conventional MIS-TLIF and ULIF group were 52.3 years and 55.1 years, respectively ( $p=0.016$ ). The male to female ratio was similar in both groups, 0.66 on conventional MIS TLIF group and 0.8 on ULIF group. There also similar BMI characteristics on both groups. The most common fusion level is L4-5 (Table 1).

Pre-operative back and leg VAS, ODI, and SF-36 were similar in both groups. All patients had a back VAS mean of 5.6, leg VAS mean of 4.5, mean ODI of 64.3, all SF-36 score showed no statistic difference in both groups. Postoperatively, the ULIF group significantly showed better back VAS (mean 2.7) directly post op $(p=0.032)$ and after 3 months $(p=0.046)$, but on the 6and 12-month follow-ups, the p-value was not significant (Table 2).

Leg VAS was similar on both groups. The p-value on directly post op, 3, 6 and 12 months were $0.700,0.773$, 0.603 and 0.067 , respectively. All the leg VAS in both groups showed improvement along the way. The leg pain improved significantly especially on the 6th month post operation. The mean VAS on ULIF group was 1.8 and on the conventional MIS-TLIF group was 2.1 (Table 2). The majority of the patients showed only slight paresthesia as a residual symptom.

The mean of ODI score of all patients was $65 \%$ which showed moderate disability on the pre-operative period. The ODI score improved significantly on both groups with $\mathrm{p}=0.0012$ and 0.0013 for the conventional MIS TLIF and ULIF respectively. The comparison between both groups on 3-, 6- and 12-months post op showed no statistical difference (Table 3). The SF-36 result also showed a comparable outcome between both groups (Table 4).

The fusion rate at the 12-month follow-up were $93.3 \%$ in the conventional MIS TLIF and $92.7 \%$ in the ULIF group ( $\mathrm{p}=0.067)$. Most of the patients in both groups had grade III or IV fusion (Figure 7). On the conventional MIS TLIF group, there were 2 patients with asymptomatic posterior cage migration. Those patients were observed for any associated symptoms.

There were 3 patients observed with dural tear in the ULIF group, the tear was covered with dural patch instead of primary repair, with no post operation neurologic deficit. Two patients in the conventional MIS TLIF group developed post-operative infection. Both patients underwent debridement and cage removal. The pedicle screws were maintained for stability. Cage subsidence also occurred in 2 patients in conventional MIS TLIF groups.

\section{Discussion}

The conventional open surgery for spinal stenosis with instability still remains the gold standard for achieving a complete decompression and fusion. This type of procedure is considered to be the workhorse for the management of

Table I General Characteristic of Degenerative Spondylolisthesis Patients Underwent Operations

\begin{tabular}{|c|c|c|c|c|}
\hline & \multirow{2}{*}{$\begin{array}{l}\text { Conventional MIS-TLIF } \\
\mathrm{N}=73\end{array}$} & \multirow{2}{*}{$\begin{array}{l}\text { ULIF } \\
\mathbf{N}=72\end{array}$} & \multirow[t]{2}{*}{ Mean Difference (Cl 95\%) } & \multirow[t]{2}{*}{ P-value } \\
\hline & & & & \\
\hline Age, years-means (SB) & $52.3(6.13)$ & $55.1(5.12)$ & $4.5(-1.9-10.9)$ & 0.160 \\
\hline Sex & & & & 0.130 \\
\hline Male & $28(37.5)$ & $26(62.5)$ & & \\
\hline Female & $45(63.2)$ & $46(36.8)$ & & \\
\hline Body Mass Index (BMI), kg/m² -means (SB) & $24.8(3.42)$ & $23.6(3.67)$ & $1.2(-1.2-3.7)$ & 0.319 \\
\hline BMI Category & & & & 0.494 \\
\hline Normal & $39(40.0)$ & $40(60.0)$ & & \\
\hline Overweight & $30(66.7)$ & $29(33.3)$ & & \\
\hline Obese & $4(57.1)$ & $3(42.9)$ & & \\
\hline Smoking History & & & & 0.227 \\
\hline Yes & II (36.4) & $17(63.6)$ & & \\
\hline No & $62(58.3)$ & $55(41.7)$ & & \\
\hline Operation Level & & & & 0.586 \\
\hline L3-4 & $10(11.42)$ & $8(10.52)$ & & \\
\hline L4-5 & $48(65.7 I)$ & $56(81.57)$ & & \\
\hline L5-SI & $15(22.85)$ & 8 (7.89) & & \\
\hline
\end{tabular}


Table 2 Comparison of Visual Analog Scale Between Conventional MIS TLIF and ULIF

\begin{tabular}{|c|c|c|c|c|}
\hline & Conventional MIS TLIF N=73 & ULIF $\mathbf{N}=72$ & Mean Difference (Cl 95\%) & P-value \\
\hline \multirow{2}{*}{\multicolumn{5}{|c|}{$\begin{array}{l}\text { Visual analog scale } \\
\text { Back }\end{array}$}} \\
\hline & & & & \\
\hline Pre operation & 5.4 & 5.7 & $0(-1-1)$ & 0.708 \\
\hline \multicolumn{5}{|l|}{ Post operation } \\
\hline Directly & 3.9 & 2.7 & $0(-I-I)$ & 0.032 \\
\hline 3 months & 2.4 & 1.9 & $0(-1-1)$ & 0.046 \\
\hline 6 months & 1.6 & 1.7 & $0(-1-1)$ & 0.660 \\
\hline 12 months & 0.9 & 0.8 & $0(0-1)$ & 0.386 \\
\hline \multicolumn{5}{|l|}{ Leg } \\
\hline Pre operation & 4.5 & 4.6 & $0(-1-1)$ & 0.909 \\
\hline \multicolumn{5}{|l|}{ Post operation } \\
\hline Directly & 4.6 & 4.6 & $0(0-I)$ & 0.700 \\
\hline 3 months & 3.6 & 3.7 & $0(0-1)$ & 0.773 \\
\hline 6 months & 1.8 & 2.1 & $I(0-I)$ & 0.603 \\
\hline 12 months & 0.8 & 0.7 & I (0-2) & 0.067 \\
\hline
\end{tabular}

Table 3 Comparison of ODI Between Conventional MIS TLIF and ULIF

\begin{tabular}{|l|l|l|l|l|}
\hline & Conventional MIS TLIF & ULIF & Mean Difference (CI 95\%) & P value \\
\cline { 2 - 3 } & $\mathbf{N = 7 3}$ & $\mathbf{N}=\mathbf{7 2}$ & & \\
\hline $\begin{array}{l}\text { Oswestry Disability Index } \\
\text { Pre operation }\end{array}$ & $62(56.5-70.5)$ & $60(52-63.5)$ & $6(-2-14)$ & 0.103 \\
$\begin{array}{l}\text { Post operation } \\
\text { Directly }\end{array}$ & $54(37-49)$ & $41(49-60.5)$ & $11(-3-17)$ & 0.045 \\
3 months & $16(5.5-14.5)$ & $12(8-32.5)$ & $-4(-12-2)$ & 0.232 \\
6 months & $12(4-12.5)$ & $8(4-19)$ & $-2(-8-4)$ & 0.483 \\
I2 months & $8(1.5-10)$ & $6(3.5-17.5)$ & $-3(-10-2)$ & 0.184 \\
\hline
\end{tabular}

degenerative lumbar conditions. However, this is not without disadvantages: Muscle injury which could lead to posterior tension band disruption; Epidural fibrosis that could cause restenosis; Intra-operative blood loss and a long hospital stay which results in long functional recovery and expensive patient and hospital care. Minimally invasive spine surgery (MISS) has been developed to counter such problems. ${ }^{4,14}$

The development of MISS is very extensive in the last 2 decades. Evolving from simple discectomy, decompression only procedure and fusion procedure using air-based medium surgery until the very recently water-based medium surgery. ${ }^{5,9}$ The conventional MIS-interbody fusion, either standard posterior lumbar interbody fusion (PLIF) or transforaminal lumbar interbody fusion (TLIF), provides a 360degree fusion using a dorsal muscle-sparing approach through tubular retractor system. End-plate preparation and interbody cages insertion are achieved through the tube using customized surgical instruments. The TLIF technique allows interspace preparation with less neural retraction than the more medial exposure by the PLIF technique. Thus, it has become more popular than MIS-PLIF. ${ }^{15}$

Later on, the water-based endoscopic procedure regain popularity due to easier bleeding control and clearer image (compared with the conventional MIS and air-based surgery). The better mobility of the endoscope and instrument creates a wider working space and a better evaluation of decompression and fusion bed preparation (in spinal fusion surgery). ${ }^{4,14}$ ULIF takes advantage of endoscopic system which preserves the paravertebral muscle and the bony structures as much as possible. This technique combines endoscope and the standard working spine instrument such as the Kerrison punch, reamers, nerve retractors, etc. ULIF uses the biportal endoscopic technique which provides more mobility and bigger working space which makes easier preparation for fusion procedure. 


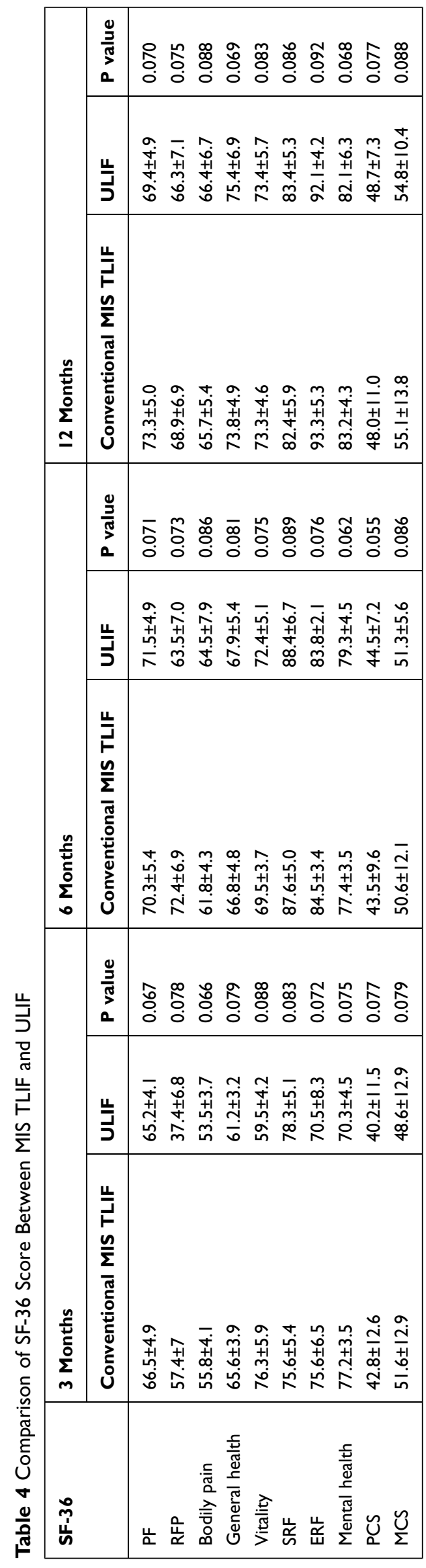

In this study, the result of ULIF was comparable with the conventional MIS-TLIF as described by Park et al. ${ }^{10}$ The result also supports the evidence that ULIF has a significantly better short-term improvement compared to the conventional MIS-TLIF. The conventional MISTLIF still has higher post-operative back pain compared with the ULIF procedure. This is probably due to more muscle injury in the conventional MIS-TLIF. The ULIF procedure also has a better visualization for the lateral recess and foraminal area without the need to increase tissue dissection as in the conventional MIS-TLIF procedure. In the conventional MIS-TLIF, bilateral approach is sometimes needed to achieve adequate decompression.

Endoscopic spine procedure which seems easy in the literature has its own learning curve. ${ }^{16-18}$ The biportal endoscopic technique needs triangulation because of a separate working portal and viewing portal just like arthroscopy. Thus, surgeons who are familiar with arthroscopy can adapt more easily. The technique also requires single-handed instrument handling which causes delicate procedures to become more complex to execute and complications such as dural tear and nerve root injury could occur, especially for less-experienced surgeons.

End-plate preparation is important in the interbody fusion procedure. The endoscopic procedure enables the operator to view the intervertebral space directly and remove the remaining nucleus and annulus after blind removal. ${ }^{10}$ Bleeding from end-plates is the sign of complete preparation. ${ }^{5,9,19}$ The capacity of viewing bony bleeding with other procedures such as conventional MISTLIF, open TLIF, OLIF, and ALIF is virtually impossible due to limited working space. Surgeons usually estimate the completeness of the preparation by feeling the rough surface of the end-plates. In order to achieve a solid fusion, a bone graft consisting of an autograft from the lamina and facet and demineralized bone matrix allograft is inserted through a special funnel into the disc space. ${ }^{20}$

The biportal endoscopic spine surgery is not without disadvantages, during the process several issues were encountered. Dural tears, even though rare, happened in 5 cases with severe flavum ligament thickening. We put a dural patch directly over the torn dura without primary repair. The patient healed uneventfully without neurological deficit. ${ }^{21}$ Epidural hematoma is another complication that might happen during an endoscopic procedure, especially a procedure with quite a lot of bony work. In our study, there were 3 cases of postoperative epidural 


\section{Radiological Outcome of Lumbar Interbody Fusion}

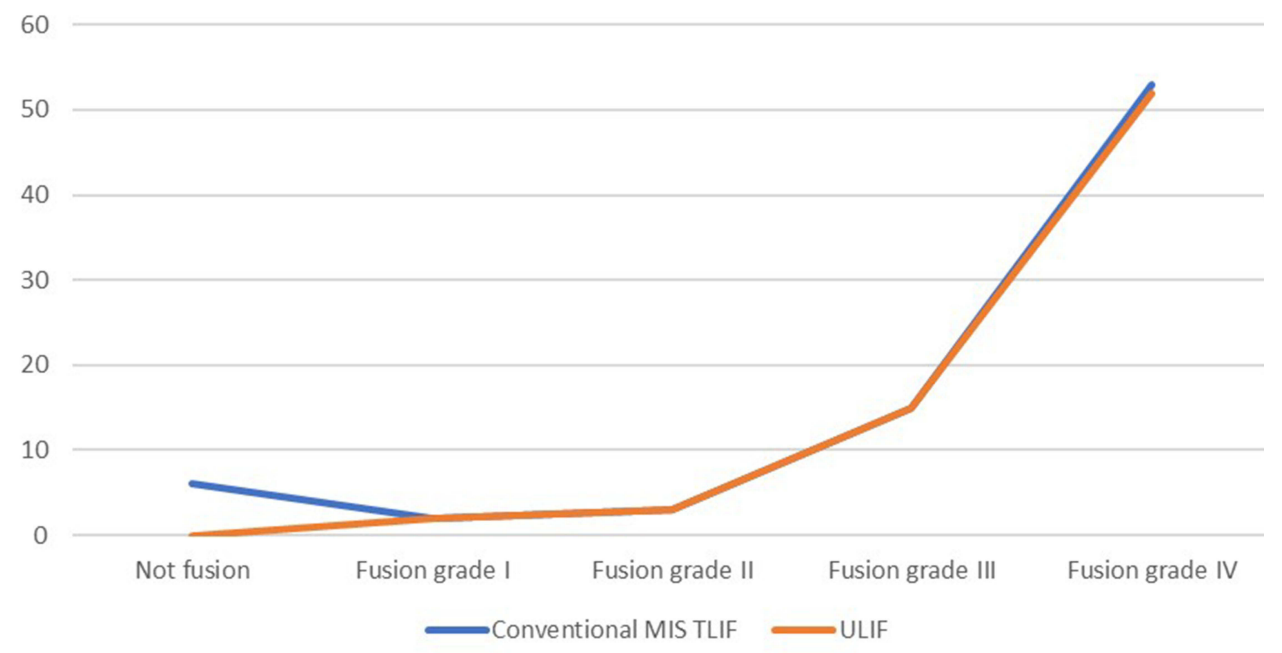

Figure 7 Fusion rate comparison showed no significant difference between ULIF group (orange line) and the conventional MIS-TLIF group (blue line).

hematoma. This is most likely due to oozing from the bone that is trapped under the intact posterior tension band which leads to increased pressure inside the epidural space. The epidural hematoma is usually resolved by itself. However, it may cause post-operative pain and discomfort. $^{22}$

Other possible complication of ULIF that might occur later is the new onset of radicular pain (after pain-free period) due to instability pain related to the implant subsidence, migration, or new pain generators (i.e., bony and scar tissue formation behind the interbody fusion cage and bulging of the remaining annulus fibrosus). ${ }^{23}$ Improvements of the implant have been made to overcome the risk of subsidence and migration problems seen with the conventional fusion cages. ${ }^{9,24}$ No recurrent radicular pain or implant failure that were observed in both clinical and radiological follow-up of ULIF group in our study. Otherwise, this complication occurred in the conventional MIS-TLIF group.

The limitation of this study is that we did not take sagittal balance into account. Some literature suggests that sagittal balance parameters abnormality has a significant role in the degeneration of adjacent segments after lumbar fusion. ${ }^{25}$ Further studies which include these parameters and a longer follow-up time are needed to evaluate the procedure thoroughly.

\section{Conclusion}

The unilateral biportal endoscopic procedure gives another option in achieving nerve decompression and bony fusion.
Our study showed that this procedure had a comparable long-term outcome and a significantly better back pain VAS reduction in short-term follow up compared to the conventional MIS-TLIF. Thus, with further improvement, ULIF can be the next future standard in managing degenerative lumbar spine conditions.

\section{Abbreviations}

ULIF, Unilateral Biportal Endoscopic Lumbar Interbody Fusion; VAS, Visual analog scale; ODI, Oswestry Disability Index; MIS-TLIF, minimally invasive lumbar interbody fusion; SAP, Superior Articular Process; IAP, Inferior Articular Process.

\section{Data Sharing Statement}

There is no other supporting data.

\section{Ethics Approval and Consent to Participate}

This research had already been approved by ethical committee in Fatmawati General Hospital Jakarta. The privacy and personal identity information of all participants were protected and written informed consent was obtained from all participants.

\section{Consent for Publication}

This manuscript does not contain any individual person data. The co-authors consented to the publication of this article. 


\section{Funding}

This research did not receive any specific grant from funding agencies in the public, commercial, or not-forprofit sectors.

\section{Disclosure}

The authors declare that they have no conflicts of interest for this work.

\section{References}

1. Derman PB, Albert TJ. Interbody fusion techniques in the surgical management of degenerative lumbar spondylolisthesis. Curr Rev Musculoskelet Med. 2017;10(4):530-538. doi:10.1007/s12178-0179443-2

2. Gokaslan ZL. A brief history of endoscopic spine surgery. Neurosurg Focus. 2016;40. doi:10.3171/2015.11.FOCUS15429

3. Birkenmaier C, Komp M, Leu HF, Wegener B, Ruetten S. The current state of endoscopic disc surgery: review of controlled studies comparing full- endoscopic procedures for disc herniations to standard procedures. Pain Physician. 2013;16:335-344. doi:10.36076/ ppj.2013/16/335

4. Kim M, Lee S, Kim HS, Park S, Shim SY, Lim DJ. A comparison of percutaneous endoscopic lumbar discectomy and open lumbar microdiscectomy for lumbar disc herniation in the Korean: a meta-analysis. Biomed Res Int. 2018;2018:1-8. doi:10.1155/2018/9073460

5. Jin M, Zhang J, Shao H, Liu J, Huang Y. Percutaneous transforaminal endoscopic lumbar interbody fusion for degenerative lumbar diseases: a consecutive case series with mean 2-year follow-up. Pain Physician. 2020;23(2):165-174.

6. Liu C, Zhou Y. Percutaneous endoscopic lumbar discectomy and minimally invasive transforaminal lumbar interbody fusion for massive lumbar disc herniation. Clin Neurol Neurosurg. 2019;176:19-24. doi:10.1016/j.clineuro.2018.10.017

7. Kolcun JPG, Brusko GD, Basil GW, Epstein R, Wang MY. Endoscopic transforaminal lumbar interbody fusion without general anesthesia: operative and clinical outcomes in 100 consecutive patients with a minimum 1-year follow-up. Neurosurg Focus. 2019;46(4):1-5. doi:10.3171/2018.12.FOCUS18701

8. Ahn Y, Youn MS, Heo DH. Endoscopic transforaminal lumbar interbody fusion: a comprehensive review. Expert Rev Med Devices. 2019;16(5):373-380. doi:10.1080/17434440.2019.1610388

9. León JFR, Ardila ÁS, Rugeles Ortíz JG, et al. Standalone lordotic endoscopic wedge lumbar interbody fusion (LEW-LIF ${ }^{\mathrm{TM}}$ ) with a threaded cylindrical peek cage: report of two cases. J Spine Surg. 2020;6(S1):S275-S285. doi:10.21037/jss.2019.06.09

10. Park MK, Park SA, Son SK, Park WW, Choi SH. Correction to: clinical and radiological outcomes of unilateral biportal endoscopic lumbar interbody fusion (ULIF) compared with conventional posterior lumbar interbody fusion (PLIF): 1-year follow-up. Neurosurg Rev. 2019;42(763):10-1007. doi:10.1007/s10143-019-01131-2

11. Yang Y, Liu ZY, Zhang LM, et al. Microendoscopy-assisted minimally invasive versus open transforaminal lumbar interbody fusion for lumbar degenerative diseases: 5-year outcomes. World Neurosurg. 2018;116:e602-e610. doi:10.1016/j.wneu.2018.05.049
12. Kim JE, Choi DJ. Unilateral biportal endoscopic spinal surgery using a $30^{\circ}$ arthroscope for L5-S1 foraminal decompression. Clin Orthop Surg. 2018;10(4):508-512. doi:10.4055/cios.2018.10.4.508

13. Kim JE, Choi DJ, Park EJ. Clinical and radiological outcomes of foraminal decompression using unilateral biportal endoscopic spine surgery for lumbar foraminal stenosis. Clin Orthop Surg. 2018;10 (4):439-447. doi:10.4055/cios.2018.10.4.439

14. Ruetten S, Komp M, Merk H, Godolias G. Full-endoscopic interlaminar and transforaminal lumbar discectomy versus conventional microsurgical technique. Spine (Phila Pa 1976). 2008;33 (9):931-939. doi:10.1097/BRS.0b013e31816c8af7

15. Theodotou CB, Wang MY. Minimally invasive transforaminal lumbar interbody fusion. Semin Spine Surg. 2021;33(3):1-6. doi:10.1016/j. semss.2021.100888

16. Hsu HT, Chang SJ, Yang SS, Chai CL. Learning curve of full-endoscopic lumbar discectomy. Eur Spine J. 2013;22 (4):727-733. doi:10.1007/s00586-012-2540-4

17. Choi CM, Chung JT, Lee SJ, Choi DJ. How I do it? Biportal endoscopic spinal surgery (BESS) for treatment of lumbar spinal stenosis. Acta Neurochir (Wien). 2016;158(3):459-463. doi:10.1007/s00701015-2670-7

18. Anichini G, Landi A, Caporlingua F, et al. Lumbar endoscopic microdiscectomy: where are we now? An updated literature review focused on clinical outcome, complications, and rate of recurrence. Biomed Res Int. 2015;2015:1-14. doi:10.1155/2015/417801

19. Heo DH, Son SK, Eum JH, Park CK. Fully endoscopic lumbar interbody fusion using a percutaneous unilateral biportal endoscopic technique: technical note and preliminary clinical results. Neurosurg Focus. 2017;43(2):1-6. doi:10.3171/2017.5.FOCUS17146

20. Gatam AR, Gatam L, Tobing SDL. Comparison of clinical and radiological outcomes of lumbar interbody fusion using a combination of hydroxyapatite and demineralized bone matrix and autografts for lumbar degenerative spondylolisthesis. Asian Spine J. 2017;11(5):706-714. doi:10.4184/asj.2017.11.5.706

21. Park HJ, Kim SK, Lee SC, Kim W, Han S, Kang SS. Dural tears in percutaneous biportal endoscopic spine surgery: anatomical location and management. World Neurosurg. 2020;136:e578-e585. Elsevier Inc. doi:10.1016/j.wneu.2020.01.080

22. Kim JE, Choi DJ, Kim MC, Park EJ. Risk factors of postoperative spinal epidural hematoma after biportal endoscopic spinal surgery. World Neurosurg. 2019;129:e324-e329. doi:10.1016/j. wneu.2019.05.141

23. Lewandrowski KU, Ransom NA, Yeung A. Subsidence induced recurrent radiculopathy after staged two-level standalone endoscopic lumbar interbody fusion with a threaded cylindrical cage: a case report. J Spine Surg. 2020;6(2):S286-S293. doi:10.21037/ jss.2019.09.25

24. Lewandrowski KU. Surgical technique of endoscopic transforaminal decompression and fusion with a threaded expandable interbody fusion cage and a report of 24 cases. J Spine. 2018;07(02). doi: $10.4172 / 2165-7939.1000409$

25. Le Huec JC, Faundez A, Dominguez D, Hoffmeyer P, Aunoble S. Evidence showing the relationship between sagittal balance and clinical outcomes in surgical treatment of degenerative spinal diseases: a literature review. Int Orthop. 2015;39(1):87-95. doi:10.1007/ s00264-014-2516-6 


\section{Publish your work in this journal}

Orthopedic Research and Reviews is an international, peer-reviewed, open access journal that focusing on the patho-physiology of the musculoskeletal system, trauma, surgery and other corrective interventions to restore mobility and function. Advances in new technologies, materials, techniques and pharmacological agents are particularly welcome. The manuscript management system is completely online and includes a very quick and fair peer-review system, which is all easy to use. Visit http://www.dovepress.com/testimonials.php to read real quotes from published authors.

Submit your manuscript here: https://www.dovepress.com/orthopedic-research-and-reviews-journal 